\title{
Enhancing subdural effusions mimicking acute subdural hematomas following angiography and endovascular procedures: report of 2 cases
}

\author{
Carlos A. Zamora, MD, PhD, and Doris D. Lin, MD, PhD \\ Division of Neuroradiology, The Russell H. Morgan Department of Radiology and Radiological Science, Johns Hopkins \\ University School of Medicine, Baltimore, Maryland
}

Hyperdense enhancing subdural effusion due to contrast extravasation has been recently described as a potential mimicker of acute subdural hematoma following a percutaneous coronary procedure. Herein, the authors report on 2 patients who presented with subarachnoid hemorrhage from ruptured cerebral aneurysms and who developed enhancing subdural effusions mimicking acute subdural hematomas after angiography and endovascular coil placement. In 1 case, the subdural effusions completely cleared but recurred after a second angiography. CT attenuation values higher than expected for blood, as well as the evolution of the effusions and density over time, allowed for differentiation of enhancing subdural effusions from acute subdural hematomas.

http://thejns.org/doi/abs/10.3171/2014.10.JNS142172

KEY WORDS subarachnoid hemorrhage; subdural effusion; subdural hematoma; aneurysm; rupture; mimic; vascular disorders; trauma

$\mathrm{A}$ CASE of extravasation of contrast material into the subdural space with development of hyperdense effusions has been reported recently as a potential mimicker of acute subdural hematoma following angiography.$^{14}$ In that study, the authors describe the case of a patient who developed hyperdense subdural effusions after a percutaneous coronary intervention.

We describe 2 patients who presented with subarachnoid hemorrhage secondary to ruptured aneurysms and who developed hyperdense subdural effusions after angiography. These mimicked acute subdural hematomas but had resolved on short-term follow-up examination. In 1 case, the effusions completely resolved on follow-up CT performed the next day and recurred after repeat angiography. In both patients there was a clear temporal relationship with the injection of intraarterial contrast. To the best of our knowledge, this is the first instance of this phenomenon described in the setting of subarachnoid hemorrhage or aneurysmal rupture.

\section{Case Reports}

Case 1

History and Examination

This 65-year-old man presented with a 2-day history of increasing confusion and agitation associated with nausea and vomiting. There was no history of trauma. On physical examination, the patient followed basic commands and showed normal strength without focal neurological deficits (Hunt and Hess Grade III). Noncontrast head CT demonstrated subarachnoid hemorrhage predominantly in the suprasellar cistern and right sylvian fissure and a small amount of intraventricular blood (Grade 4 on the modified Fisher scale). Findings were highly suspicious for a ruptured aneurysm. Additionally, the patient had small bilateral hypodense subdural collections without significant mass effect on the brain parenchyma (Fig. 1).

\section{Operation and Postoperative Course}

The patient underwent angiography, which demonstrated a 5.1-mm ruptured aneurysm arising from the anterior communicating artery. There was no evidence of arterial extravasation into the subdural space. Balloon-assisted coil embolization was performed without complications. The follow-up head CT scan performed 5 hours after coil embolization demonstrated increased density within the previously hypodense subdural collections, which were also slightly larger. The average Hounsfield unit (HU) value within the collections was 35 , compared with 18 before angiography. At this point, it was not clear whether this

ABBREVIATION HU = Hounsfield unit(s).

SUBMITTED September 16, 2014. ACCEPTED October 30, 2014.

INCLUDE WHEN CITING Published online April 24, 2015; DOI: 10.3171/2014.10.JNS142172.

DISCLOSURE The authors report no conflict of interest concerning the materials or methods used in this study or the findings specified in this paper. 
increased density and mild enlargement was secondary to contrast extravasation or interval hemorrhage into the subdural space. Repeat head CT 1 day later demonstrated resolution of hyperdensity within the subdural collections and decreased size with an appearance comparable to the baseline study (Fig. 1). The patient was extubated on the same day, continued to improve neurologically, and was discharged 19 days after admission on physical and occupational therapy.

\section{Case 2}

History and Examination

This 52-year-old woman presented with a several-hour history of confusion and increased unsteadiness. Her medical history included stroke, metastatic cervical cancer, congestive heart failure, and chronic kidney disease, and she was being treated with clopidogrel on a long-term basis. On physical examination, she was alert and oriented and had residual left-side weakness as well as a pronator drift, which were not different from her baseline (Hunt and Hess Grade III). A noncontrast CT scan of the head revealed subarachnoid hemorrhage centered in the suprasellar cistern (Grade 3 on the modified Fisher scale). There were no subdural or parenchymal hematomas (Fig. 2).

\section{Operation and Postoperative Course}

The patient underwent cerebral angiography, which revealed a $2.4-\mathrm{mm}$ aneurysm at the origin of the right superior hypophyseal artery, determined to be the source of hemorrhage. There was no evidence of arterial extravasation into the subdural space. Endovascular treatment was performed by means of balloon-assisted coil embolization, which achieved successful stagnation of contrast material within the aneurysm. CT examination of the head 4 hours after intervention demonstrated new hyperdense subdural collections along the cerebral hemispheres and interhemispheric fissure (Fig. 2), with an appearance consistent with acute subdural hematomas given their increased density and associated mass effect. However, the average CT attenuation measured $97 \mathrm{HU}$, higher than expected for blood. A follow-up CT study performed the next day showed complete resolution of the collections (Fig. 2). On the same day, several hours after the follow-up CT, the patient also underwent uneventful coil embolization of an inferior pancreatic artery aneurysm diagnosed during the course of hospitalization. CT of the head on the following day again demonstrated hyperdense subdural collections that were similar to the ones identified after cerebral aneurysm embolization 2 days before, with an even higher average $\mathrm{HU}$ value of 124 . These demonstrated progressive resolution on head CT studies 2 and 6 days later (Fig. 2). The patient's hospital course was, however, further complicated by multifocal infarcts due to vasospasm, septic shock, disseminated intravascular coagulation, and pulmonary embolism. She died 36 days after admission.

\section{Discussion}

While the effusions in the cases presented in this paper had the appearance of acute subdural hematomas, their rapid evolution over serial imaging as well as CT attenuation values indicate that they were related to enhancing effusions filling the subdural space as opposed to hemorrhage. Before the breakdown of red blood cells, freshly extravasated blood is hyperattenuated on CT due to its high electron density. However, iodinated contrast material still displays significantly higher density on CT (range 91-274 $\mathrm{HU}$, mean $155 \mathrm{HU}$ ) compared with that of fresh blood (range $28-82 \mathrm{HU}$, mean $54 \mathrm{HU}){ }_{1}^{18}$ due its more favorable $\mathrm{K}$-shell binding energy. Our second case showed attenuation values that were higher than blood, particularly on the second follow-up study, which showed recurrence of the subdural effusions. However, these differences may not be as obvious visually and could easily be misinterpreted, unless the possibility of an enhancing effusion is specifically suspected. A caveat to the above is the fact that contrast medium may be diluted in the cerebrospinal fluid or in previously existing effusions and thus may not appear as dense, which is probably what occurred in the first case. In that patient, while there were both a visual and a measurable increase in the density of the small existing effusions, these were not as dense or conspicuous as in the second case. Nevertheless, the measured density was most probably higher than expected for blood, particularly if taking into account that blood itself may also be diluted in fluid and lose some of its density. Additionally, the rapid clearance of hyperattenuation on the follow-up examination the next day would not be easily explained by acute subdural hemorrhage.

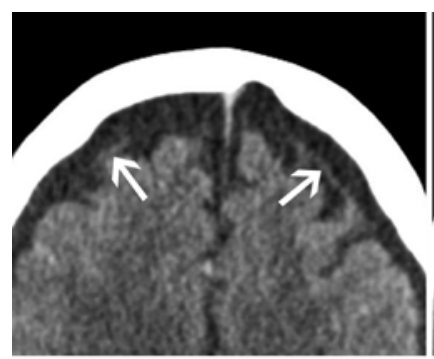

BASELINE

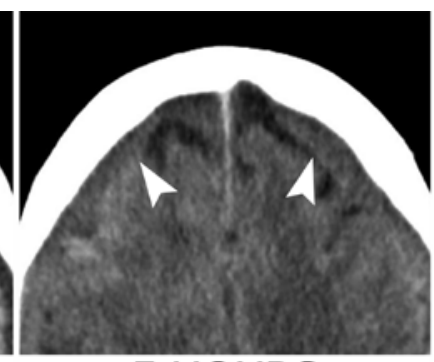

5 HOURS

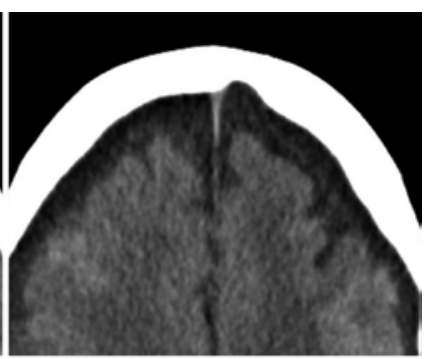

DAY 2

FIG. 1. Case 1. This 65-year-old man presented with a 2-day history of increasing confusion and agitation. The unenhanced baseline CT image obtained at initial presentation demonstrates small bilateral hypodense subdural effusions with displacement of cortical vessels (arrows). The image from a follow-up CT scan performed 5 hours after coil embolization shows increased density of the subdural effusions and also a slight increase in size, most notably along the left frontal convexity, where there is increased displacement of cortical vessels (arrowheads). The effusions have decreased in attenuation and slightly decreased in size as seen in the CT image obtained on Day 2, with an appearance similar to baseline. 


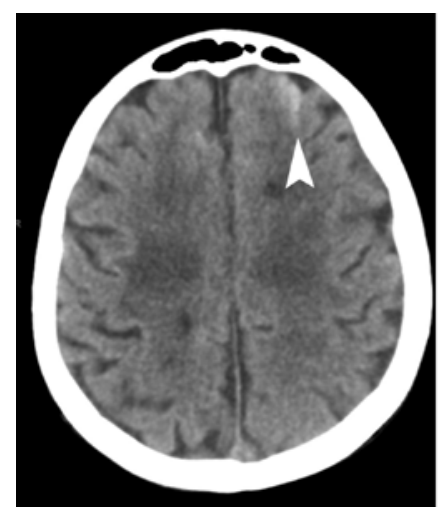

BASELINE

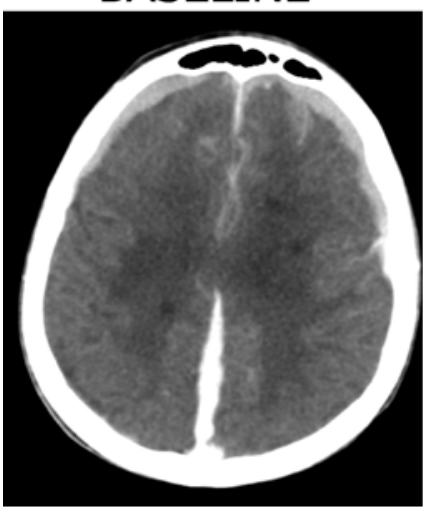

DAY 4

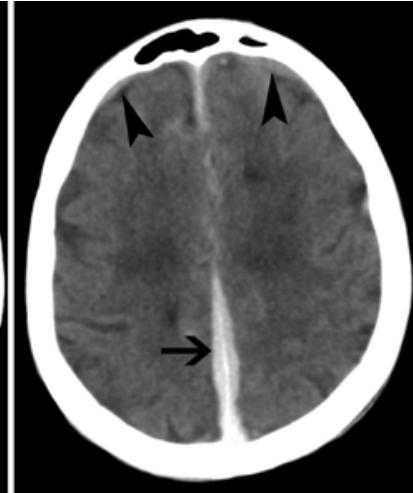

DAY 2

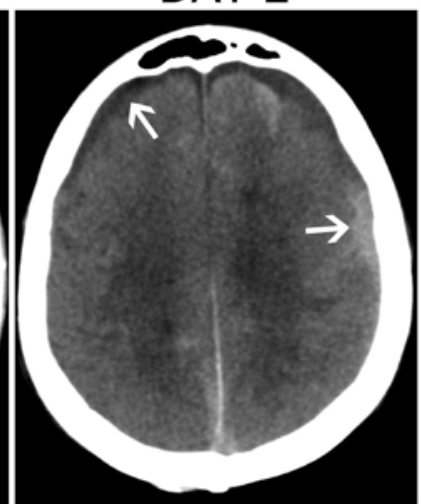

DAY 6

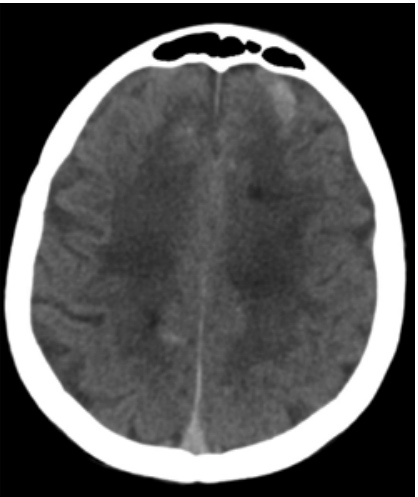

DAY 3

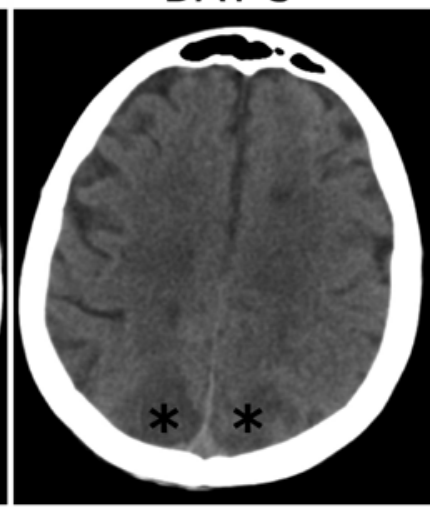

DAY 9

FIG. 2. Case 2. This 52-year-old woman presented with a several-hour history of confusion and increased falls. Baseline unenhanced CT shows a small amount of subarachnoid blood (white arrowhead) without subdural hematomas or effusions. The image from the follow-up study obtained the next day, 4 hours after endovascular coiling of a superior hypophyseal aneurysm, shows a hyperdense subdural effusion along the falx cerebri (black arrow) and small subdural effusions along the frontal lobes bilaterally (black arrowheads). These nearly completely resolved on Day 3 , except for trace residual effusion along the right frontal lobe. The image from a head CT study obtained the next day (Day 4) demonstrates recurrence of the hyperdense collections after repeat angiography for endovascular embolization of a pancreatic aneurysm. The images from studies obtained on Days 6 and 9 show progressive resolution of the collections (arrows). Hypodensities in the occipital lobes on Day 9 correspond to developing infarcts due to vasospasm (asterisks).

There are a few possible explanations for contrast enhancement of the subdural space. One possibility is that increased dural endothelial permeability with extravasation of contrast material occurred. It has been demonstrated that subarachnoid hemorrhage triggers a number of pathophysiological changes in the microvasculature, including a prominent neuroinflammatory response and disruption of the blood-brain barrier. ${ }^{16}$ A range of inflammatory mediators are released during subarachnoid hemorrhage, including interleukin (IL)-1 $\beta$, IL-6, and tumor necrosis factor (TNF) $-\alpha$, among others, which modulate the cerebral endothelium of the blood-brain barrier, increasing its permeability. ${ }^{1,7}$ Experimental studies in animal models have indicated that such inflammatory processes can occur even in regions remote from the bleeding site or the subarachnoid space. ${ }^{13}$ A different pattern of contrast leak, into the subarachnoid rather than subdural space, was described in a case report involving a patient who underwent coil embolization for a ruptured intracranial aneurysm, presumably also due to disruption of the blood-brain barrier but postulated to relate to the use of nonionic contrast. ${ }^{17}$

Another possibility is diffusion of contrast material due to increased membrane permeability. Various MRI stud- ies have reported diffusion of gadolinium into acute and chronic subdural hematomas or effusions. ${ }^{6,10,11}$ Chronic subdural hematomas and effusions develop an outer neomembrane featuring a high capillary surface area and a high transfer rate constant, which allows extravasation of plasma components into the subdural space and also results in increased volume of the effusion. ${ }^{9,11}$ A prior electron microscopy study has shown degeneration of capillary endothelial cells in the capsules of chronic subdural hematomas, with multiple cytoplasmic protrusions and fenestrations and lack of a basement membrane, which usually covers the basal surface of the normal endothelium. ${ }^{15}$ Such findings suggest increased permeability. This could potentially explain why there was enhancement in the first case presented, which had small baseline, presumably chronic, subdural effusions. However, it would not account for the presentation of our second case, where no subdural effusions or collections were present.

Traumatic subdural hygromas also have vascularized, permeable outer neomembranes with more fenestrations. ${ }^{5}$ However, as opposed to subdural effusions, hygromas have a direct communication with the subarachnoid space through an arachnoid tear. Subdural hygromas are there- 
fore expected to enhance together with the subarachnoid space, as has been previously demonstrated..$^{10}$ Our cases are not consistent with subdural hygromas, as we observed isolated enhancement of the subdural space in both patients.

In both cases, no new or associated neurological symptoms were documented. Both followed a self-limited course and spontaneously resolved very rapidly, without requirement of therapy or intervention. It remains elusive, however, what truly accounted for the occurrence, since this phenomenon has not been observed or reported following a rather commonly performed procedure and intracranial event.

The case reported by Rennert and Hamer showed enhancing subdural effusions remarkably similar to both cases presented here. ${ }^{14}$ Although their patient did not initially have a known intracranial abnormality or undergo an intracranial procedure, she did suffer a cardiac arrest and demonstrated evidence of global hypoxic injury on neuroimaging. The endothelium is directly in contact with blood and exquisitely responsive to oxygen tension and thus hypoxia, presumably accounting for the disruption of blood-brain barrier and increased permeability. Prior studies have also shown a proinflammatory response in the presence of hypoxia, which can be exacerbated by ischemia-reperfusion injury. ${ }^{4,8}$ These factors and potentially other mechanisms of blood-brain barrier disruption may have contributed to the enhancing subdural effusions.

Finally, subdural hemorrhage caused by intracranial aneurysms is rare but well documented, postulated to occur by mechanisms leading to rupture of the arachnoid membrane. ${ }^{12}$ Even more rarely encountered is spontaneous subdural hematoma due to active arterial extravasation into the subdural space that can be depicted by angiography..$^{2,3}$ These entities are typically associated with neurological symptoms and require surgical intervention, and are therefore important to distinguish from a "benign" contrast extravasation as a result of increased vascular or membrane permeability that is self-limited. Whereas careful attention to CT attenuation values and evolution of the abnormality over serial imaging can be helpful discriminators as in the 2 cases presented here, issues may arise when reviewing film images, where CT values may have not been measured. This could be particularly problematic with outside studies or when prior CT images are not readily available.

In summary, the occurrence of enhancing subdural effusions after different angiographic procedures is documented in 2 patients with ruptured cerebral aneurysms. These may mimic subdural hematomas, potentially resulting in unwarranted intervention. Attention to CT attenuation values and evolution of the effusions on short-term follow-up imaging can be helpful discriminators.

\section{References}

1. Abbott NJ: Inflammatory mediators and modulation of blood-brain barrier permeability. Cell Mol Neurobiol 20:131-147, 2000

2. Akioka N, Fukuda O, Takaba M, Kameda H, Saito T, Endo S: Clinical investigation of acute spontaneous subdural hematoma cases. J Stroke Cerebrovasc Dis 16:109-113, 2007
3. Dalfino JC, Boulos AS: Visualization of an actively bleeding cortical vessel into the subdural space by CT angiography. Clin Neurol Neurosurg 112:737-739, 2010

4. Flamant L, Toffoli S, Raes M, Michiels C: Hypoxia regulates inflammatory gene expression in endothelial cells. Exp Cell Res 315:733-747, 2009

5. Hasegawa M, Yamashima T, Yamashita J, Suzuki M, Shimada S: Traumatic subdural hygroma: pathology and meningeal enhancement on magnetic resonance imaging. Neurosurgery 31:580-585, 1992

6. Kanamalla US, Baker KB, Boyko OB: Gadolinium diffusion into subdural space: visualization with FLAIR MR imaging. AJR Am J Roentgenol 176:1604-1605, 2001

7. Maruo N, Morita I, Shirao M, Murota S: IL-6 increases endothelial permeability in vitro. Endocrinology 131:710-714, 1992

8. Michiels C, Arnould T, Remacle J: Endothelial cell responses to hypoxia: initiation of a cascade of cellular interactions. Biochim Biophys Acta 1497:1-10, 2000

9. Mori K, Adachi K, Cho K, Ishimaru S, Maeda M: Quantitative kinetic analysis of blood vessels in the outer membranes of chronic subdural hematomas. Neurol Med Chir (Tokyo) 38:697-703, 1998

10. Mori K, Maeda M: Delayed magnetic resonance imaging with GdD-DTPA differentiates subdural hygroma and subdural effusion. Surg Neurol 53:303-311, 2000

11. Mori K, Mitsuoka H, Cho K, Tajima A, Maeda M: Rate constant of gadolinium (Gd)-DTPA transfer into chronic subdural hematomas. Neurol Res 18:126-134, 1996

12. Oh SY, Kwon JT, Park YS, Nam TK, Park SW, Hwang SN: Clinical features of acute subdural hematomas caused by ruptured intracranial aneurysms. J Korean Neurosurg Soc 50:6-10, 2011

13. Prunell GF, Svendgaard NA, Alkass K, Mathiesen T: Inflammation in the brain after experimental subarachnoid hemorrhage. Neurosurgery 56:1082-1092, 2005

14. Rennert J, Hamer OW: Large subdural effusions after angiography mimicking acute subdural hematoma. J Comput Assist Tomogr 34:249-250, 2010

15. Sato S, Suzuki J: Ultrastructural observations of the capsule of chronic subdural hematoma in various clinical stages. J Neurosurg 43:569-578, 1975

16. Tso MK, Macdonald RL: Subarachnoid hemorrhage: a review of experimental studies on the microcirculation and the neurovascular unit. Transl Stroke Res 5:174-189, 2014

17. Uchiyama Y, Abe T, Hirohata M, Tanaka N, Kojima K, Nishimura H, et al: Blood brain-barrier disruption of nonionic iodinated contrast medium following coil embolization of a ruptured intracerebral aneurysm. AJNR Am J Neuroradiol 25:1783-1786, 2004

18. Willmann JK, Roos JE, Platz A, Pfammatter T, Hilfiker PR, Marincek B, et al: Multidetector CT: detection of active hemorrhage in patients with blunt abdominal trauma. AJR Am J Roentgenol 179:437-444, 2002

\section{Author Contributions}

Conception and design: both authors. Drafting the article: Zamora. Critically revising the article: both authors. Reviewed submitted version of manuscript: both authors. Approved the final version of the manuscript on behalf of both authors: Lin.

\section{Correspondence}

Doris D. Lin, Division of Neuroradiology, The Russell H. Morgan Department of Radiology and Radiological Science, Johns Hopkins University School of Medicine, 601 N. Caroline St., Baltimore, MD 21287. email: ddmlin@jhmi.edu. 